\title{
Análisis Experimental y Numérico de la Trayectoria de Propagación de Fisuras por Fatiga Utilizando XFEM
}

\author{
David R. Berrios y Rosendo Franco \\ Grupo INACOM/Aula PUCP-CIMNE, Sección Ing. Mecánica, Dpto. de Ingeniería, Pontificia Universidad \\ Católica del Perú, Av. Universitaria 1801, Lima 32-Perú \\ (e-mail: dberrios@pucp.edu.pe,rofranco@pucp.edu.pe)
}

Recibido May. 17, 2017; Aceptado Jul. 17, 2018; Versión final Oct. 15, 2017, Publicado Oct. 2018

\begin{abstract}
Resumen
En este trabajo se presentan los resultados experimentales de la trayectoria de propagación de fisuras por fatiga en cinco probetas de acero de alta resistencia. Fue necesario definir la geometría de las probetas e implementar un procedimiento para realizar los ensayos, ya que no existen normas para la evaluación de la trayectoria de propagación de fisuras por fatiga. La simulación numérica se desarrolló en el entorno del software ANSYS MECHANICAL APDL, utilizando tecnología XFEM. Se implementaron tres criterios para determinar la dirección de propagación: el criterio de la Máxima Tensión Tangencial (MTS), el criterio de la Máxima Tasa de Liberación de Energía (MERR) y el criterio de la Mínima Densidad de Energía de Deformación (SED). Los tres criterios implementados arrojan resultados prácticamente idénticos, alcanzando un coeficiente de determinación $R^{2}$ igual a 0.997 , respecto a los resultados experimentales, indicando con ello que hay una fuerte correspondencia entre los resultados experimentales y numéricos.
\end{abstract}

Palabras clave: mecánica de fractura; factor de intensidad de tensiones; propagación de fisuras por fatiga; XFEM; LEFM

\section{Experimental and Numerical Analysis of Fatigue Crack Propagation Path Using XFEM}

\begin{abstract}
In this work the experimental results of the fatigue crack propagation path on five high strength steel specimens are presented. It was necessary to define the geometry of the test specimens and to implement a procedure to perform such tests, since no standards for the evaluation of the fatigue crack propagation path exist. The numerical simulation was developed in ANSYS Mechanical APDL environment using XFEM technology. Three criteria to determine the propagation direction were implemented: the Maximum Tangential Stress criterion (MTS), the Maximum Energy Release Rate criterion (MERR) and the Minimum Strain Energy Density criterion (SED). The three implemented criteria yield practically identical results, reaching a coefficient of determination $R^{2}$ equal to 0.997 with respect to the experimental results, indicating that a strong correspondence between the experimental and numerical results exist.
\end{abstract}

Keywords: fracture mechanics; stress intensity factors; fatigue crack propagation; XFEM; LEFM 


\section{INTRODUCCIÓN}

El comportamiento de un elemento fisurado sometido a cargas cíclicas es un campo de la ingeniería estudiado por la Mecánica de la Fractura. Predecir la trayectoria de la propagación de fisuras por fatiga ha sido el punto de atención de muchos investigadores, debido a que es necesario conocerla para evaluar su tamaño crítico, a partir del cual habrá una propagación súbita hasta la rotura del elemento. La Mecánica de la Fractura Elástica Lineal (LEFM) abarca el estudio de elementos fisurados cuya deformación plástica en la zona aledaña a la punta de la fisura es altamente localizada. Este comportamiento es propio de materiales frágiles, tales como los vidrios y los cerámicos. También los aceros de alta resistencia, fragilizados por tratamientos térmicos o por hidrógeno, pueden fracturarse de forma frágil. Este es el caso del material que se estudia en el presente trabajo: acero $\mathrm{W} \mathrm{N}^{\circ}$ : 1.8721 con tratamiento térmico de temple y revenido. Algunos estudios, como los de Islam (2006) y Wang et al. (2016), corroboran los efectos del fenómeno de fragilización en aceros similares.

En los últimos años muchos investigadores han puesto su atención en el estudio del crecimiento de fisuras por fatiga. Palma et al. (2010), usaron conceptos de la mecánica de la fractura y la mecánica del daño continuo, en la simulación numérica de fisuras en materiales degradados por efecto de la fragilización por hidrogeno. Mróz (2010), realizó un estudio de los modelos de crecimiento de fisuras en condiciones de cargas en modo mixto. Así mismo, Richard et al. (2014), investigaron sobre las teorías, experimentos y simulaciones del crecimiento de fisuras sometidas a modos de carga combinada. Estos autores describen varios criterios para predecir la dirección de propagación de la fisura, tres de los cuales se utilizan en el presente trabajo y se detallan más adelante. Por otra parte, Andrade et al. (2015), estudiaron los diferentes modelos de crecimiento de fisuras por fatiga, con énfasis en la ley de Paris para la evaluación de la velocidad del crecimiento de fisuras en la etapa de crecimiento estable. Otros estudios presentan la simulación del efecto de plastificación en la propagación de fisuras por fatiga en modo mixto, en probetas CT con la ayuda de un taladro desviador (Ferreño et al., 2006). Ellos evaluaron la trayectoria de propagación de la fisura utilizando criterios tradicionales para predecir la dirección de propagación. Ayatollahi et al. (2015), realizaron un estudio experimental y numérico de la influencia del taladro desviador en la propagación de fisuras, usaron la ecuación de Nasgro para el crecimiento y el criterio de la máxima tensión tangencial para la determinación de la carga equivalente para modos de carga mixto I+ll. Estos autores comprobaron un retardo del crecimiento de fisuras en probetas CT de Aluminio. Martins et al. (2016), evaluaron experimental y numéricamente el crecimiento de fisuras por fatiga para dos tipos de aceros inoxidables austeníticos, el AISI 316L y un acero de alta resistencia al Cr-Mn. Los autores usaron métodos ópticos en la evaluación del crecimiento en probetas delgadas CT y el FEM para el cálculo de los factores de intensidad de tensiones en modos I y III. Shariati et al. (2016), realizaron un análisis experimental y numérico del crecimiento de fisuras por fatiga en recipientes a presión de paredes gruesas de material Ck45, evaluaron la trayectoria de propagación de fisuras semielípticas y aplicaronn la ley de Paris en la evaluación del crecimiento. En estos análisis usaron el método de los elementos de contorno tridimensionales para la simulación numérica. Silva et al. (2017), realizaron un estudio experimental para determinar los factores de intensidad de tensiones en un acero estructural, usaron probetas CT con un taladro desviador para producir modos de carga mixto. En este trabajo aplicaron la técnica de correlación digital de imágenes para la detección de trayectorias producidas por fatiga, usaron la ley de Paris y el criterio de Tanaka para evaluar el crecimiento en modos I+II.

Irwin (1957), planteó las relaciones que existen entre la energía, las deformaciones y las tensiones, en el campo de LEFM, suponiendo que el proceso de propagación de la fisura se desarrolla elásticamente. Este autor obtuvo también la expresión para determinar la disminución de la energía potencial del sistema $G$ en función de los factores de intensidad de tensiones $K_{I}$ y $K_{I I}$, a medida que se propaga la fisura. Sus estudios permiten afirmar que en condiciones de plasticidad a pequeña escala la fisura se encuentra controlada por los tres parámetros mencionados. Este criterio no considera los micro mecanismos de fractura producidos en el vértice de la fisura, denominada la zona de proceso, que son bastante complejos y siguen siendo objeto de investigación. Los análisis de mecánica de fractura se pueden realizar por diferentes métodos, como son: métodos experimentales, métodos analíticos y métodos numéricos. El Método de los Elementos Finitos (FEM) es uno de los métodos numéricos más potentes para solucionar problemas de ingeniería. Sin embargo, el FEM es menos eficiente para modelar problemas que involucran discontinuidades o singularidades, siendo necesario utilizar procedimientos de mallado especiales para capturar la singularidad en el extremo de la fisura, lo cual es costoso computacionalmente. En el trabajo de Silva et al. (2017) se aprecia que han utilizado una malla con un alto grado de refinamiento en toda la zona de propagación de la fisura desde el inicio, tomando como base los resultados experimentales obtenidos. Ayatollahi et al. (2015), describen un procedimiento para estimar el crecimiento de fisuras por fatiga, el cual requiere una técnica de remallado en la punta de la fisura en cada incremento de crecimiento de la fisura. Asimismo, Branco et al. (2015), resaltan la importancia de las técnicas de remallado adaptativo basadas en FEM que han usado diferentes autores para modelar el crecimiento de fisuras. En cambio, el Método de los Elementos Finitos Extendidos (XFEM) permite modelar adecuadamente discontinuidades en movimiento, facilitando la simulación del crecimiento y propagación de fisuras, sin necesidad de regeneración de malla ni refinamiento extremo en la región aledaña 
a la fisura (Gonzales, 2010). Sharma et al. (2016), usaron XFEM para simular la vida por fatiga en una aleación de aluminio y alúmina, bajo condiciones de cargas cíclicas combinadas, usaron el criterio MTS en la determinación de la dirección de propagación.

Para determinar los factores de intensidad de tensiones es necesario evaluar la energía de deformación elástica producida alrededor de la fisura, para esto se puede aplicar el método de la Integral de Interacción a partir de una solución numérica obtenida por XFEM. La tecnología XFEM se ha aplicado en la simulación del crecimiento de fisuras tridimensionales por fatiga, utilizando la ley de Paris y el criterio del máximo esfuerzo principal (Pathak et al. 2013). Asimismo, Bahloul et al. (2017), usaron el XFEM para evaluar los factores de intensidad de tensiones y determinar el crecimiento de fisuras por fatiga en 2D. Estos autores consideraron la influencia de las tensiones residuales, producidas en la punta de la fisura, en la evaluación de la velocidad de crecimiento en aluminio 7075-T6. Por su parte, Bergara et al. (2017), realizaron un análisis experimental y numérico del crecimiento de fisuras semielípticas por fatiga en una viga sometida a flexión, usando el XFEM para la evaluación de los factores de intensidad de tensiones.

A diferencia de algunas investigaciones anteriormente mencionadas, el presente trabajo tiene como finalidad analizar la trayectoria de propagación de fisuras sometidas a cargas cíclicas, haciendo uso de ensayos experimentales y de simulación numérica. Para realizar los ensayos experimentales se diseñaron y fabricaron probetas especiales, tomando en consideración las propiedades mecánicas del material y las cargas a aplicar para producir la fatiga. Para realizar la simulación numérica se evaluaron tres criterios reportados en la literatura para predecir la dirección de propagación de la fisura: MTS, MERR y SED. Para ello se desarrollaron algoritmos y se implementaron macros en el entorno del software ANSYS MECHANICAL APDL, utilizando la tecnología XFEM. Los resultados obtenidos con los procedimientos numéricos son comparados con los resultados experimentales, con la finalidad de validar los algoritmos y modelos desarrollados.

\section{ENSAYOS EXPERIMENTALES}

Para realizar los ensayos experimentales de propagación de fisuras se caracterizó previamente el material de las probetas. Luego se diseñó una probeta típica y se fabricaron cinco probetas para los ensayos de propagación de fisuras, con diferente ubicación del denominado taladro desviador. Finalmente se estableció un procedimiento para ejecutar los ensayos de propagación de fisuras.

\section{Caracterización del material}

En la Tabla 1 se muestran los resultados del análisis de composición química del acero W N: 1.8721, del cual se fabricaron las probetas para el estudio. Estos datos fueron adquiridos con el Espectrómetro de Emisión Óptica Q8, Maguellan Bruker. Además, mediante análisis metalográfico se pudo identificar la microestructura martensítica del material, obtenida mediante tratamiento térmico de temple y revenido.

Tabla 1: Composición química del material de la probeta, Acero W №: 1.8721

\begin{tabular}{|c|c|c|c|c|c|c|c|c|c|c|}
\hline Composición Química & $\mathrm{C}$ & $\mathrm{Si}$ & $\mathrm{Mn}$ & $\mathrm{P}$ & $\mathrm{S}$ & $\mathrm{Cr}$ & $\mathrm{Mo}$ & $\mathrm{Ni}$ & $\mathrm{Al}$ & $\mathrm{Co}$ \\
\hline \multirow{4}{*}{ Acero W No: 1.8721} & 0.211 & 0.367 & 0.819 & 0.0161 & 0.0007 & 0.316 & 0.028 & 0.112 & 0.062 & 0.0023 \\
\cline { 2 - 12 } & $\mathrm{Cu}$ & $\mathrm{Nb}$ & $\mathrm{Ti}$ & $\mathrm{V}$ & $\mathrm{W}$ & $\mathrm{Sn}$ & $\mathrm{As}$ & $\mathrm{Zr}$ & $\mathrm{Ce}$ & $\mathrm{Te}$ \\
\cline { 2 - 11 } & 0.023 & 0.002 & 0.0033 & $<0.0032$ & $<0.0015$ & 0.0018 & 0.001 & 0.0013 & 0.0046 & $<0.001$ \\
\hline
\end{tabular}

De forma similar, se realizaron los ensayos para obtener las propiedades mecánicas del material. Se realizaron 2 ensayos de tracción según la norma ASTM A-370 (2013), para obtener la resistencia a la tracción, el límite de fluencia y el módulo de elasticidad. Para determinar la dureza Rockwell C se realizó el ensayo de dureza según la norma ASTM E-18 (2014), siendo realizadas 8 indentaciones en diferentes partes de la probeta. Finalmente, para obtener la tenacidad a la fractura del material, se realizó el ensayo correspondiente según la norma ASTM E-399 (2013). Para este fin se ensayaron 5 probetas, de las cuales 4 fueron validadas según la norma. Los resultados de estos ensayos se pueden apreciar en la Tabla 2.

Tabla 2: Propiedades Mecánicas del material de la probeta, Acero W N: 1.8721

\begin{tabular}{|l|c|l|l|}
\hline Ensayos Mecánicos / Norma & Parámetro & Máquina de ensayo & \multicolumn{1}{c|}{ Observaciones } \\
\hline \multirow{2}{*}{$\begin{array}{l}\text { Resistencia a la tracción / } \\
\text { ASTM A-370 }\end{array}$} & $1586 \mathrm{MPa}$ & $\begin{array}{l}\text { Máquina de tracción } \\
\text { SP600 Zwick/Roell }\end{array}$ & Transversal a la dirección de laminación \\
\cline { 2 - 4 } & $1610 \mathrm{MPa}$ & Longitudinal a la dirección de laminación \\
\hline
\end{tabular}


Tabla 2 (continuación)

\begin{tabular}{|c|c|c|c|}
\hline \multirow{2}{*}{$\begin{array}{l}\text { Limite convencional de } \\
\text { fluencia / ASTM A-370 }\end{array}$} & $1469 \mathrm{MPa}$ & & Transversal a la dirección de laminación \\
\hline & $1490 \mathrm{MPa}$ & & Longitudinal a la dirección de laminación \\
\hline $\begin{array}{l}\text { Módulo de elasticidad / ASTM } \\
\text { A-370 }\end{array}$ & $206 \mathrm{GPa}$ & & \\
\hline Dureza / ASTM E-18 & $52 \mathrm{HRC}$ & $\begin{array}{l}\text { Durómetro Rockwell } \\
\text { Instron 2002T } \\
\end{array}$ & En estado natural (temple - revenido) \\
\hline $\begin{array}{l}\text { Tenacidad a la fractura del } \\
\text { material Kıc / ASTM E-399 }\end{array}$ & $3194 \mathrm{MPa} \sqrt{\mathrm{mm}}$ & $\begin{array}{l}\text { Máquina de tracción Z250 } \\
\text { Zwick/Roell }\end{array}$ & Ensayo validado bajo la ASTM E-399 \\
\hline
\end{tabular}

\section{Diseño y fabricación de las probetas}

El dimensionamiento de las probetas obedece a la intención del estudio, que es obtener trayectorias largas y curveadas, que proporcionen datos suficientes para evaluar numéricamente la eficacia de diferentes criterios de dirección de propagación de fisuras. Debido a esto se optó por una probeta tipo doble viga en voladizo con sección variable (TDCB), incluyendo un taladro desviador. Las dimensiones establecidas (en $\mathrm{mm}$ ) se indican en la Fig. 1 a) y las coordenadas $(X, Y)$ del centro del taladro desviador, según el sistema coordenado de la misma figura, se muestran en la Tabla 3. El espesor máximo de las probetas está sujeto a las dimensiones de las mordazas de fijación, no debiendo ser muy delgadas para evitar altas cargas de flexión sobre los pines. El espesor de las probetas utilizadas en este estudio es de $12 \pm 0.2 \mathrm{~mm}$. La rugosidad superficial en todas las superficies de las probetas es de $1.6 \mu \mathrm{m}$, excepto en las caras laterales que son rectificadas y alcanza $0.8 \mu \mathrm{m}$. En la Fig. 1 b) se observa una probeta montada, luego del ensayo de propagación.

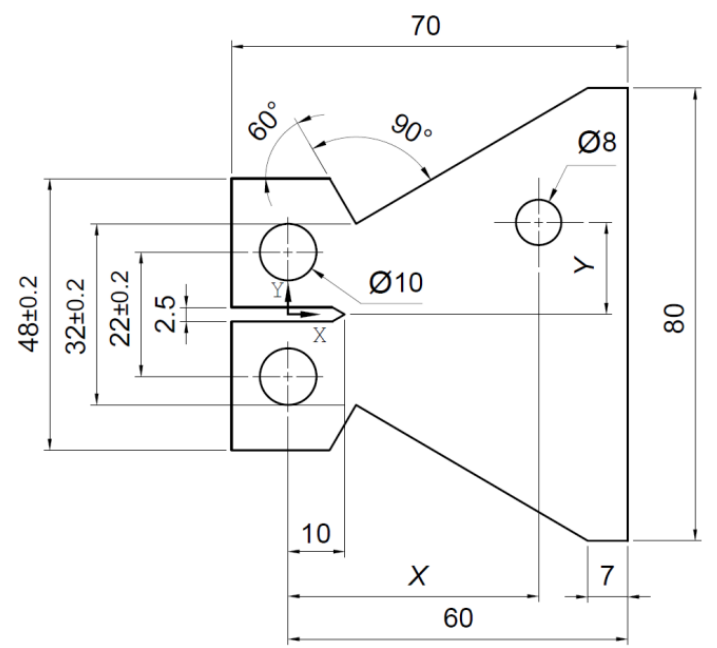

a)

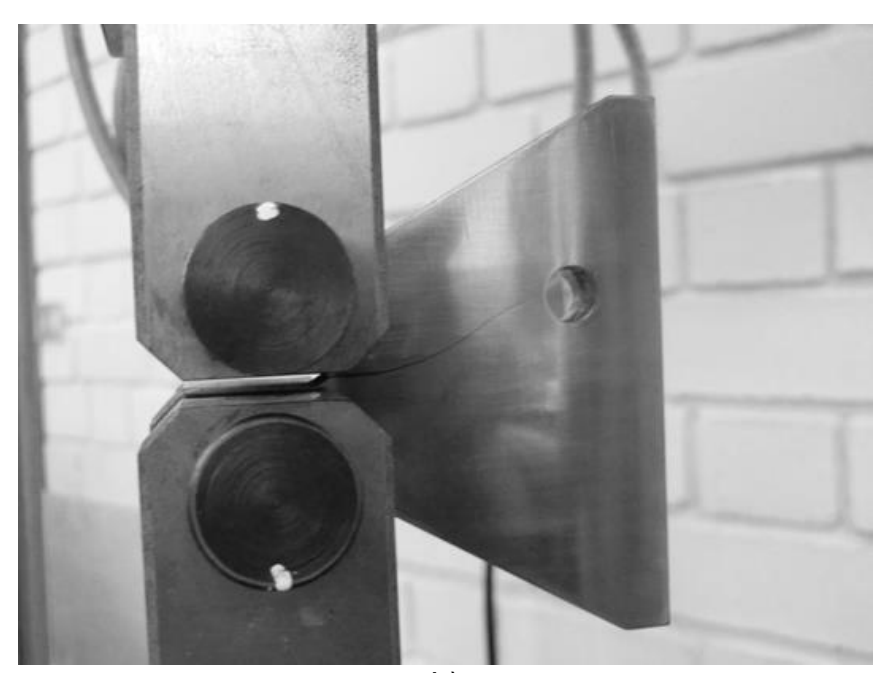

b)

Fig. 1: Probeta para ensayos de propagación, a) plano de fabricación, b) montaje en máquina de ensayos

El proceso de fabricación de las probetas es importante para obtener buenos resultados en los posteriores ensayos, por lo cual se describe a continuación cómo se debe realizar dicho proceso:

$1^{\circ}$ Se fabrica la probeta por procesos de mecanizado con arranque de viruta. El material debe encontrarse en estado revenido, con una dureza de $25 \mathrm{HRC}$ aproximadamente, esto facilita el proceso de manufactura. No se elaboran aquí la entalla ni el taladro desviador.

$2^{\circ}$ Se realiza un tratamiento térmico de temple y revenido, para obtener una dureza entre $51 \mathrm{HRC}$ y $53 \mathrm{HRC}$. Luego de este tratamiento el acero alcanza una microestructura martensítica.

$3^{\circ}$ Se fabrica la entalla de $20 \mathrm{~mm}$ de profundidad y $2.5 \mathrm{~mm}$ de ancho por proceso de electroerosión por hilo, según se indica en la Fig. $1 \mathrm{a}$ ), con un ángulo de $60^{\circ}$ y un radio no mayor a $0.1 \mathrm{~mm}$ en la punta de la entalla. Seguidamente se realiza el rectificado de las caras laterales mediante un mecanizado por abrasión y luego se realiza un pulido manual con lijas de agua, aplicando consecutivamente las lijas número $80,120,220,400,800$ y 1200.

$4^{\circ}$ Se genera una pre-fisura por fatiga de $8 \mathrm{~mm}$ de longitud, tomando en cuenta los procedimientos y consideraciones establecidos en la norma ASTM E-399.

$5^{\circ}$ Se elabora el taladro desviador de $8 \mathrm{~mm}$ de diámetro. Para este proceso se debe usar una broca carburada debido a la alta dureza del material. 
Para este trabajo la pre-fisura se generó en un equipo Vibrophore AMSLER 150 HFP 5100-Zwick/Roell, utilizando una carga cíclica de amplitud constante. Cabe señalar que las pre-fisuras de las cinco probetas no son idénticas entre sí y en general no están perfectamente alineadas con el plano de simetría. Esto puede ocurrir por errores inherentes al proceso de fabricación y por imperfecciones en la microestructura del material.

\section{Ensayos de propagación de fisuras}

En la literatura revisada no se han encontrado procedimientos para realizar ensayos de propagación de fisuras, orientados al estudio de la trayectoria. En tal sentido fue necesario establecer un procedimiento de ensayo que consiste en estimar las cargas adecuadas para inducir el crecimiento de la fisura y a la vez evitar la ruptura súbita de la probeta. Para ello se establecieron tres tramos con diferentes magnitudes de la carga aplicada, el primer tramo presenta la carga más alta y luego va disminuyendo, como se puede observar en los resultados de los ensayos de propagación de la fisura, mostrados en la Tabla 3 . Los ensayos de propagación fueron realizados en el mismo equipo Vibrophore AMSLER 150 HFP 5100-Zwick/Roell, utilizado para la generación de la pre-fisura. Los parámetros tabulados son: la carga máxima aplicada $(P)$, la razón de carga mínima y carga máxima $(R)$, el número de ciclos de carga aplicada en cada etapa $(\Delta N)$ y el correspondiente incremento de crecimiento de la fisura $(\Delta a)$. El método utilizado para determinar $\Delta a$ se basa en la obtención de las imágenes del ensayo a través de un equipo de correlación digital de imágenes, luego se insertan las imágenes en un software CAD y se mide en éste el tamaño de la fisura correspondiente a cada $\Delta N$. Usando esta misma técnica se obtuvieron las coordenadas de los puntos de la trayectoria de propagación. El equipo de ensayo se programó para que se detuviera cuando la frecuencia de la carga aplicada alcanzara una caída (disminución) de $5 \mathrm{~Hz}$, debido al crecimiento de la fisura. En cada probeta analizada el ensayo finalizó cuando la fisura se propagó hasta el taladro desviador.

Tabla 3: Parámetros de ensayo y resultados de la propagación de fisuras

\begin{tabular}{|c|c|c|c|c|c|c|c|c|c|c|c|c|c|c|}
\hline \multicolumn{3}{|c|}{ Probeta } & \multicolumn{4}{c|}{ Primer tramo } & \multicolumn{3}{c|}{ Segundo tramo } & \multicolumn{3}{c|}{ Tercer tramo } \\
\hline & $\begin{array}{c}X \\
(\mathrm{~mm})\end{array}$ & $\begin{array}{c}Y \\
(\mathrm{~mm})\end{array}$ & $\begin{array}{c}P \\
(\mathrm{~N})\end{array}$ & $R$ & $\begin{array}{c}\Delta N \\
(\text { Ciclos })\end{array}$ & $\begin{array}{c}\Delta a \\
(\mathrm{~mm})\end{array}$ & $\begin{array}{c}P \\
(\mathrm{~N})\end{array}$ & $R$ & $\begin{array}{c}\Delta N \\
(\text { Ciclos })\end{array}$ & $\begin{array}{c}\Delta a \\
(\mathrm{~mm})\end{array}$ & $\begin{array}{c}P \\
(\mathrm{~N})\end{array}$ & $\begin{array}{c}\Delta N \\
(\text { Ciclos })\end{array}$ & $\begin{array}{c}\Delta a \\
(\mathrm{~mm})\end{array}$ \\
\hline 1 & 40.3 & 13.5 & 10000 & $2 / 5$ & 196558 & 16.0 & 7000 & $3 / 7$ & 97413 & 5.6 & - & - & - & - \\
\hline 2 & 45.9 & 10.4 & 9000 & $1 / 3$ & 187384 & 15.0 & 7000 & $3 / 7$ & 144487 & 8.0 & 5000 & $3 / 5$ & 100000 & 3.0 \\
\hline 3 & 45.4 & 10.2 & 9000 & $1 / 3$ & 198682 & 15.0 & 7500 & $1 / 3$ & 84188 & 10.0 & 6000 & $1 / 3$ & 39857 & 4.5 \\
\hline 4 & 46.1 & 9.8 & 12000 & $1 / 3$ & 124924 & 12.6 & 9000 & $1 / 3$ & 65174 & 11.7 & 6000 & $1 / 3$ & 11729 & 2.6 \\
\hline 5 & 46.0 & 10.2 & 12000 & $1 / 3$ & 135692 & 12.5 & 9000 & $1 / 3$ & 62196 & 11.4 & 7500 & $1 / 3$ & 5318 & 2.2 \\
\hline
\end{tabular}

\section{FORMULACIÓN XFEM}

La esencia del XFEM es la incorporación de funciones especiales en el campo de solución de desplazamientos, conocidas como funciones de enriquecimiento, lo que implica incorporar nuevos términos a la aproximación estándar del FEM (Fernandez y Domínguez, 2010). Moës et al. (1999), plantearon un procedimiento para considerar el campo discontinuo en las caras de la fisura, adaptando la función generalizada Heaviside. Por otra parte, en la LEFM los problemas presentan un comportamiento singular en la punta de la fisura. Para capturar la solución singular se incluyen elementos especiales que representan la singularidad, esto se logra con funciones de enriquecimiento del espacio de aproximación (Gonzales, 2010). Fernández y Domínguez (2010), y Gonzales (2010), sostienen que el enriquecimiento de las funciones base se debe realizar en forma local, solo es necesario afectar aquellos nodos de la malla que intercepten el dominio de interés.

El campo de los desplazamientos en XFEM está dado por la ecuación (1). El primer término de esta ecuación representa los desplazamientos del FEM clásico, el segundo término representa el campo discontinuo a través de los flancos de la fisura y el tercer término representa el enriquecimiento para el campo singular de la punta de la fisura (Moës, 1999; Gonzales, 2010; Fernández y Domínguez, 2010).

$$
u^{h}(x)=\sum_{i \in I} u_{i} N_{i}(x)+\sum_{j \in J} b_{j} N_{j}(x) H(x)+\sum_{k \in K} N_{k}(x)\left(\sum_{l=1}^{4} a_{k}^{l} B_{l}\left(r, \theta_{E}\right)\right)
$$

Donde: $N_{i}(x), N_{j}(x)$, y $N_{k}(x)$ son las funciones de forma asociadas a los nodos del elemento; $x$ representa las coordenadas del punto del dominio; $I$ es el conjunto de nodos estándar; $J$ es el conjunto de nodos asociados 
a los elementos diseccionados por la fisura; $K$ es el conjunto de nodos pertenecientes a los elementos del extremo de la fisura; $u_{i}, b_{j}$ y $a_{k}^{l}$ representan los grados de libertad correspondiente a los desplazamientos; $H(x)$ es la función de discontinuidad definida por la ecuación (2):

$$
H(x)=\left\{\begin{array}{c}
1 \text { para } y>0 \\
-1 \text { para } y<0
\end{array}\right.
$$

$B_{l}\left(r, \theta_{E}\right)$, son las funciones de enriquecimiento en coordenadas polares $\left(r, \theta_{E}\right)$, dada por la ecuación (3). Estas funciones abarcan la solución asintótica de la punta de la fisura, donde $l$ es el nodo del elemento en la punta de la fisura.

$$
B_{l}\left(r, \theta_{E}\right)=\left\{\sqrt{r} \operatorname{sen}\left(\frac{\theta_{E}}{2}\right), \sqrt{r} \cos \left(\frac{\theta_{E}}{2}\right), \sqrt{r} \operatorname{sen}\left(\frac{\theta_{E}}{2}\right) \operatorname{sen}\left(\theta_{E}\right), \sqrt{r} \cos \left(\frac{\theta_{E}}{2}\right) \operatorname{sen}\left(\theta_{E}\right)\right\}
$$

En la propagación de fisuras mediante XFEM se usan técnicas numéricas para determinar la ubicación de la fisura. La discontinuidad que se produce en la fisura se representa mediante funciones de distancia denominadas Level Set. Stolarska (2001), implementó un algoritmo para acoplar el método denominado Level Set Method (LSM) con XFEM. De esta forma se modela el crecimiento de fisuras en 2D, incluyendo la ubicación de la punta de la fisura. Las funciones Level Set son interpoladas sobre los elementos diseccionados mediante las funciones de forma $N_{j}(x)$ asociadas al nodo $j$, según las ecuaciones (4) y (5).

$$
\begin{aligned}
& \phi_{i}(x)=\sum_{j \in J} \phi_{i j} N_{j}(x) \\
& \psi(x)=\sum_{j \in J} \psi_{j} N_{j}(x)
\end{aligned}
$$

\section{DIRECCIÓN DE PROPAGACIÓN DE LA FISURA}

Tal como se ha comentado en la introducción, en el presente estudio se analizarán los tres criterios más conocidos para determinar la dirección de propagación de fisuras. A continuación, se detalla cada uno de ellos.

\section{Criterio de la Máxima Tensión Tangencial (MTS)}

Erdogan y Sih (1963), analizaron la dirección de propagación en una placa plana de material frágil, con una fisura central sometida a esfuerzo cortante puro. En base a sus investigaciones, plantearon la hipótesis de que la dirección de propagación $\theta$ está en la dirección de la máxima tensión tangencial, donde el esfuerzo cortante es cero. De esta forma obtuvieron la ecuación (6), que determina el ángulo de propagación $\theta$, siendo los valores de $K_{I}$ y $K_{I I}$ los que definirán el sendero de propagación de la fisura.

$$
\left[K_{I} \operatorname{sen} \theta+K_{I I}(3 \cos \theta-1)\right]=0
$$

Según este criterio, la máxima desviación de la trayectoria para un estado de carga en modo II puro $\left(K_{I}=0\right.$ y $K_{I I} \neq 0$ ) se obtendría al sustituir esta condición en la ecuación (6), obteniéndose la ecuación (7).

$$
\cos \theta=\frac{1}{3}
$$

De la ecuación (7) se obtienen los valores $\theta_{\text {máx }}=70.5^{\circ}$ y $\theta_{\text {mín }}=-70.5^{\circ}$. Estos valores han sido corroborados por Erdogan y Sih (1963), Wu (1978b) y Mroz (2010). Bajo carga combinada la fisura propagará en una dirección limitada por estos valores extremos.

\section{Criterio de la Máxima Tasa de Liberación de Energía (MERR)}

Griffith (1921), plantea que para materiales de comportamiento frágil la fisura podría propagarse a lo largo de la dirección de máxima liberación de la energía elástica, siempre que se logre un valor crítico de esta energía. Hussain (1974), planteó expresiones paramétricas para evaluar esta energía en casos de cargas combinadas. Posteriormente Wu (1978a), comprobó los resultados de las expresiones de Hussain para diferentes casos de carga y geometría, en modelos conocidos de la mecánica de la fractura. Existen varios métodos para determinar la tasa de liberación de energía $G_{(\theta)}$, como los descritos por Bouchard et al. (2003). Uno de los 
métodos para el cálculo de los factores de intensidad de tensiones $K_{I}$ y $K_{I I}$, cuando se añade una pequeña fisura infinitesimal orientada un ángulo $\theta$, se expresa a través de las ecuaciones (8), (9) y (10) descritas por Cotterell y Rice (1980).

$$
\begin{aligned}
& G_{(\theta)}=\frac{\left(1-v^{2}\right)}{E}\left[K_{I(\theta)}^{2}+K_{I I(\theta)}^{2}\right] \\
& K_{I(\theta)}=\frac{1}{4}\left[3 \cos \left(\frac{\theta}{2}\right)+\cos \left(\frac{3 \theta}{2}\right)\right] K_{I}-\frac{3}{4}\left[\operatorname{sen}\left(\frac{\theta}{2}\right)+\operatorname{sen}\left(\frac{3 \theta}{2}\right)\right] K_{I I} \\
& K_{I I(\theta)}=\frac{1}{4}\left[\operatorname{sen}\left(\frac{\theta}{2}\right)+\operatorname{sen}\left(\frac{3 \theta}{2}\right)\right] K_{I}+\frac{1}{4}\left[\cos \left(\frac{\theta}{2}\right)+3 \cos \left(\frac{3 \theta}{2}\right)\right] K_{I I}
\end{aligned}
$$

Según este criterio, la dirección de propagación $\theta$ coincide con aquélla en la cual la tasa de liberación de energía $G_{(\theta)}$, dada por las expresiones (8), (9) y (10), se maximiza. $E$ es el módulo de elasticidad del material y $v$ es el coeficiente de Poisson. La máxima desviación de la trayectoria para un estado de carga en modo II puro se logra al derivar la ecuación (8) con respecto a $\theta$ e igualarla a cero, obteniéndose la ecuación (11).

$$
4 \cos \left(\frac{\theta}{2}\right)+9 \cos \left(\frac{3 \theta}{2}\right)=0
$$

Al resolver la ecuación (11) se obtienen los valores $\theta_{\text {máx }}=73.87^{\circ}$ y $\theta_{\text {mín }}=-73.87^{\circ}$. Palaniswamy y Knauss (1974), Wu (1978b) y Mroz (2010), obtuvieron valores de $\theta_{\text {máx }}$ y $\theta_{\min }$ usando otros métodos para determinar $G_{(\theta)}$.

Criterio de la Mínima Densidad de Energía de Deformación (SED)

Sih (1974) plantea que, en una fisura sometida a cargas en modo mixto, la dirección de propagación $\theta$ es gobernada por la densidad de energía de deformación $S$, dada por las expresiones (12), (13), (14) y (15). Valores elevados de esta densidad de energía dificultan el crecimiento de la fisura, haciendo que ésta se propague en la dirección en que la densidad de energía es mínima. $G_{T}$ es el módulo de elasticidad transversal del material; $k_{E}$ es la constante elástica, $k_{E}=3-4 v$ para el caso de deformación plana y $k_{E}=(3-v) /(1+v)$ para el caso de tensión plana.

$$
\begin{aligned}
& S=a_{11} \frac{K_{I}^{2}}{\pi}+2 a_{12} K_{I} K_{I I}+a_{22} \frac{K_{I I}^{2}}{\pi} \\
& a_{11}=\frac{1}{16 G_{T}}\left[(1+\cos \theta)\left(k_{E}-\cos \theta\right)\right] \\
& a_{12}=\frac{1}{16 G_{T}} \operatorname{sen} \theta\left[2 \cos \theta-\left(k_{E}-1\right)\right] \\
& a_{22}=\frac{1}{16 G_{T}}\left[\left(k_{E}+1\right)(1-\cos \theta)+(1+\cos \theta)(3 \cos \theta-1)\right]
\end{aligned}
$$

La máxima desviación de la trayectoria, según este criterio, se obtiene al derivar la densidad de energía de deformación $S$ con respecto a $\theta$ e igualarla a cero, obteniéndose la ecuación (16) para el caso de tensión plana y la ecuación (17) para el caso de deformación plana.

$$
\begin{aligned}
& \cos \theta=\frac{1-v}{3(1+v)} \\
& \cos \theta=\frac{1-2 v}{3}
\end{aligned}
$$


La expresión (17) se puede corroborar de Sih y Macdonald (1974). El material utilizado en el presente estudio tiene un valor de $v=0.3$ y el análisis se realiza bajo el criterio de tensión plana, obteniéndose los valores $\theta_{\text {máx }}=79.66^{\circ}$ y $\theta_{\text {mín }}=-79.66^{\circ}$. Sih (1974) y Wu (1978b) han obtenido valores de $\theta_{\text {máx }}$ y $\theta_{\text {mín }}$ para el caso de deformación plana.

\section{PROCEDIMIENTO PARA SIMULAR LA TRAYECTORIA DE PROPAGACIÓN DE LA FISURA}

Los autores desarrollaron un procedimiento iterativo para simular la trayectoria de propagación de la fisura en base a la LEFM, utilizando la formulación XFEM descrita, el método LSM y los tres criterios definidos para determinar la dirección de propagación. El procedimiento fue implementado en el entorno del software ANSYS Mechanical APDL 17.2 y el diagrama de flujo correspondiente se muestra en la Fig. 2. Las principales etapas del procedimiento se describen a continuación.

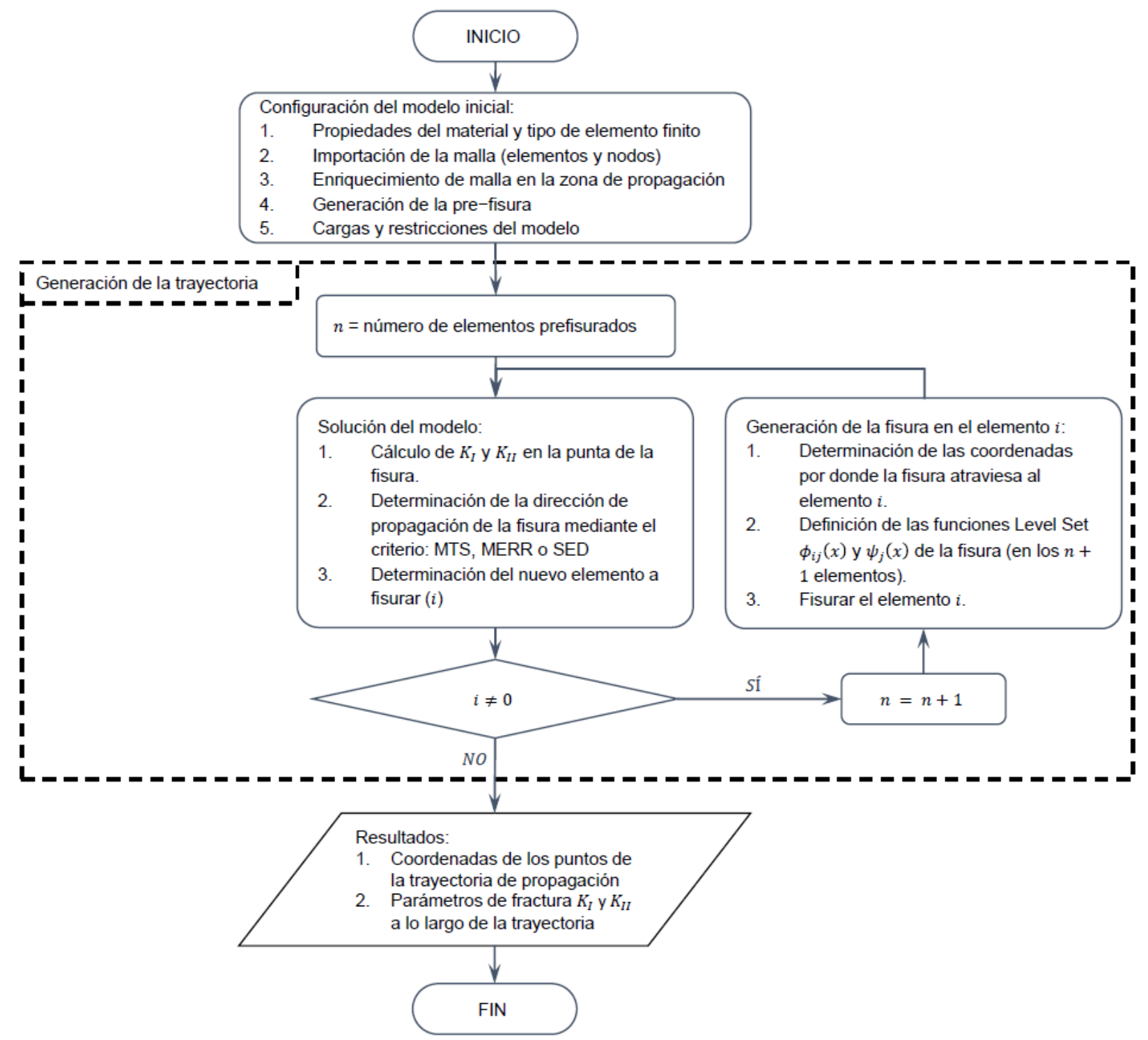

Fig. 2: Diagrama de flujo del procedimiento para la simulación de la trayectoria de propagación

\section{Configuración del modelo inicial}

Se definen las propiedades mecánicas necesarias del material: $E=206 \mathrm{GPa}$ y $v=0.3$, así como $R$ y $P$ según Tabla 3. Se crea la geometría del modelo, que coincide con la geometría de las probetas utilizadas en los ensayos experimentales, especificada en la Fig. 1. Se define el tipo de elemento finito, utilizándose en el presente estudio el cuadrilátero de primer orden (PLANE182), considerándose además un estado de tensión plana, con espesor definido (12 $\mathrm{mm}$ en todas las probetas analizadas). Se discretiza el modelo y se crea la pre-fisura inicial de $8 \mathrm{~mm}$, utilizando el procedimiento descrito más adelante, en la etapa de generación de la trayectoria de propagación. Se procura crear una pre-fisura idéntica a la generada experimentalmente. Para lograrlo se dividió la pre-fisura en varios tramos para obtener las coordenadas de sus puntos extremos, según 
el procedimiento explicado en los ensayos de propagación de fisuras. De este modo, para crear la pre-fisura en el modelo, la dirección de propagación coincide con la pendiente del tramo correspondiente, siendo un dato conocido cuando se aplica el procedimiento para crear la pre-fisura. Con el objetivo de obtener resultados adecuados es necesario generar la malla de forma controlada, suficientemente fina en la zona por donde se prevé que pase la trayectoria de la fisura. Para ello es conveniente realizar un estudio de sensibilidad de malla, hasta lograr la convergencia de los resultados de la variable de interés, que en este caso es la trayectoria de la fisura. Se establecen las restricciones de desplazamientos en el modelo y se aplica la carga, de tal modo que se pueda reproducir lo más fielmente posible la situación real de los ensayos experimentales. Para ello se define un nodo en el centro de cada agujero de sujeción, al que se acoplan los nodos del contorno del agujero correspondiente a la zona de contacto con el pin. Uno de los nodos centrales se restringe en las direcciones horizontal y vertical (nodo fijo, ubicado en la parte inferior) y el otro, donde se aplica la carga, se restringe en dirección horizontal solamente. La carga se aplica considerando los tres tramos y demás datos indicados en la Tabla 3. El modelo de análisis con el mallado, restricciones y carga aplicada se muestra en la Fig. 3.

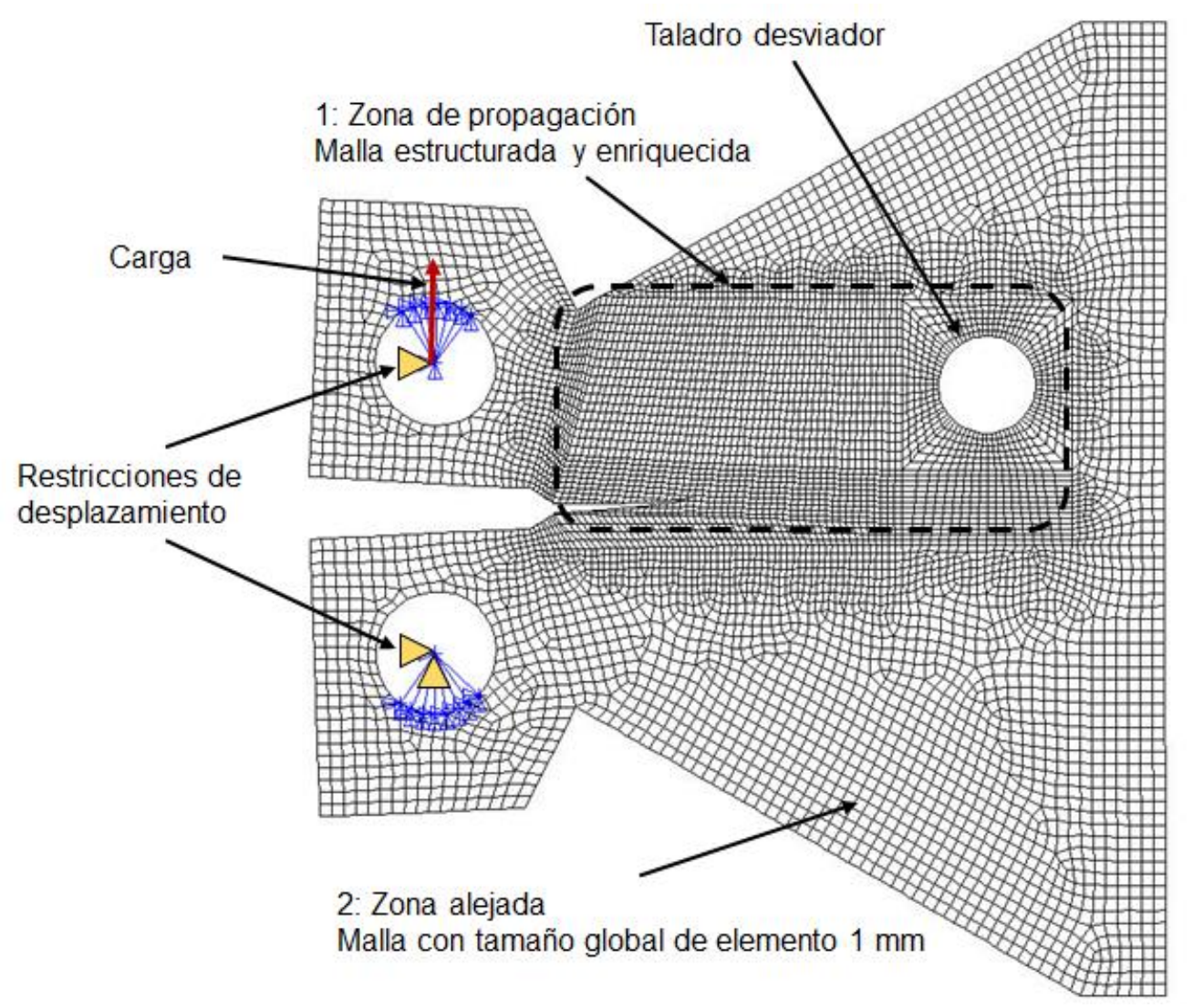

Fig. 3: Modelo de análisis.

\section{Determinación de la dirección de propagación}

En cada iteración se determinan los valores de $K_{I}$ y $K_{I I}$ en la punta de la fisura, o pre-fisura si se trata de la primera iteración. Con estos valores se elige el criterio MTS, MERR o SED, descritos en la sección anterior, y se determina la dirección de propagación de la fisura $\theta$. Este ángulo se mide en el sistema de coordenadas local $X_{L} Y_{L}$ que se define en la punta de la fisura, con el eje $X_{L}$ paralelo al último tramo de la trayectoria, como se indica en la Fig. 4 a). Los tres criterios conducen a una ecuación no lineal, solucionada en esta investigación mediante el método de la bisección en los tres casos. Cabe señalar que el software ANSYS utiliza solamente el criterio MTS, mientras que el procedimiento implementado por los autores, además de los tres criterios mencionados, permitiría incorporar otros criterios de dirección de propagación para ser evaluados.

Cuando se utiliza el método LSM la fisura puede diseccionar a un elemento una única vez. Sin embargo, cuando se determina la dirección de propagación según los criterios evaluados, podría ocurrir que la fisura tienda a propagar nuevamente por el mismo elemento, ocasionando error en la simulación. Esta situación suele presentarse cuando la dirección de la trayectoria de propagación es muy próxima a la dirección del lado del elemento que corta. Para superar esta dificultad los autores proponen realizar una corrección al ángulo de propagación, que consiste en comprobar que $\theta_{c}^{-}<\theta<\theta_{c}^{+}$. Si no cumple porque $\theta<\theta_{c}^{-}$, entonces se corrige $\theta=\theta_{c}^{-}+0.001^{\circ}$ y si no cumple porque $\theta>\theta_{c}^{+}$, entonces se corrige $\theta=\theta_{c}^{+}-0.001^{\circ}$, ver Fig. 4 a). 


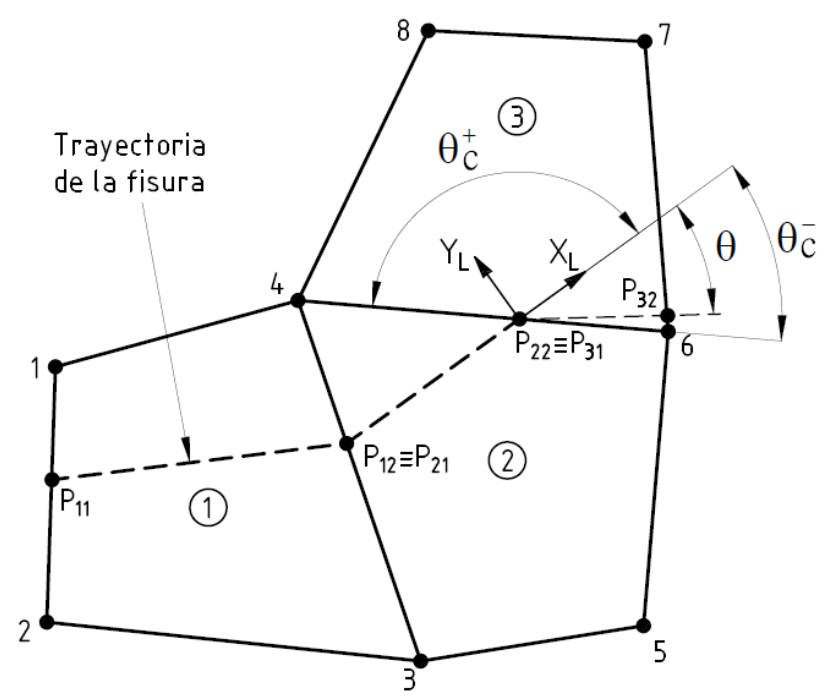

a)

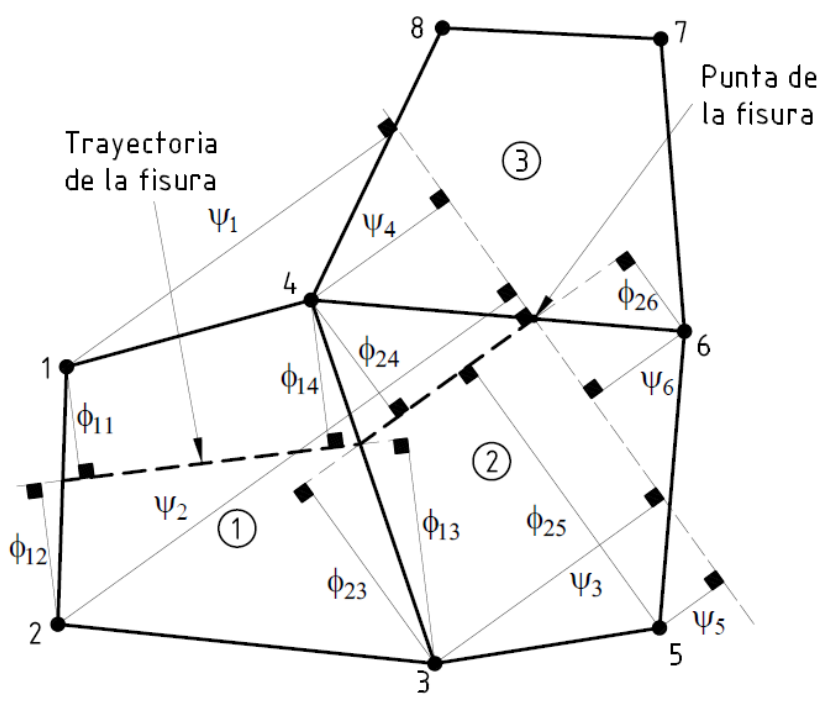

b)

Fig. 4: Parámetros para la propagación, a) dirección de propagación, b) función Level Set

\section{Generación de la trayectoria de propagación}

El crecimiento de la fisura en cada iteración alcanza a un elemento, atravesándolo de un lado a otro, por tanto, la punta de la fisura siempre estará ubicada sobre el lado de un elemento. El avance de la fisura en cada iteración depende del tamaño del elemento y de la dirección de propagación, por ejemplo, en la Fig. 4 a) el avance de la fisura, cuando se atraviesa el elemento 2, es la distancia entre los puntos $P_{12}$ y $P_{22}$. Se define la recta de propagación en el sistema de coordenadas local, con la dirección de propagación calculada $\theta$. Se determina el punto de intersección de esta recta con el lado del elemento donde propagará la fisura. En este punto se ubicará la punta de la fisura en la siguiente iteración. En el ejemplo de la Fig. 4 a), el elemento donde propagará la fisura es el número 3 y el punto de intersección es identificado como $P_{32}$.

En cada iteración $i$ es necesario determinar los parámetros de la función Level Set $\phi_{i j}(x)$ y $\psi_{j}(x)$ de todos los nodos asociados a los elementos diseccionados. El parámetro $\phi_{i j}(x)$ indica la distancia del nodo a la fisura y se define por elemento. El parámetro $\psi_{j}(x)$ indica la distancia del nodo a la punta de la fisura, paralela al último tramo de la trayectoria. En el ejemplo de la Fig. 4 b) se indican los parámetros mencionados para los nodos de los elementos diseccionados 1 y 2 .

\section{APLICACIÓN DEL PROCEDIMIENTO DESARROLLADO Y ANÁLISIS DE RESULTADOS}

Primero se realizó un análisis preliminar para calibrar el modelo y comparar los tres criterios implementados para determinar la dirección de propagación. Luego se seleccionó uno de los tres criterios para analizar todas las probetas y comparar los resultados numéricos con los experimentales.

\section{Análisis preliminar para calibrar el modelo}

Se estableció una estrategia de mallado que consistió en dividir la geometría de la probeta en dos zonas, la primera corresponde a la zona de propagación y la segunda a la zona alejada. La zona de propagación se discretizó con una malla estructurada y todos sus nodos fueron enriquecidos. En esta zona de propagación se probaron ocho tamaños diferentes de elementos $(1.0,0.8,0.6,0.4,0.3,0.2,0.1,0.075 \mathrm{~mm})$ para el análisis de sensibilidad de malla, mientras que en la zona alejada se utilizó un único tamaño $(1.0 \mathrm{~mm})$. En la Fig. 3 se pueden apreciar las dos zonas de mallado utilizadas.

El análisis de sensibilidad de malla corresponde al modelo de la probeta 3 , ya que las cinco probetas analizadas son muy similares, siendo la trayectoria de la probeta 3 la más compleja (mayor longitud y curvatura). La variable de interés, como ya se mencionó, es la trayectoria de la fisura, que depende de los factores de intensidad de tensiones $K_{I}$ y $K_{I I}$.

Para el análisis de sensibilidad estos factores fueron calculados en la punta de la pre-fisura, considerando las condiciones de contorno indicadas en la configuración del modelo inicial. En la Fig. 5 a) se muestran los resultados de la trayectoria para los ocho tamaños de elemento analizados, observándose que con tamaño $0.1 \mathrm{~mm}$ y $0.075 \mathrm{~mm}$ se alcanzan resultados adecuados, logrando que la trayectoria se desvíe hacia el taladro, tal como ocurrió experimentalmente. En la Fig. 5 b) se muestran los valores de $K_{I}$ y $K_{I I}$ en función del tamaño 
de elemento, observándose que también para los tamaños $0.1 \mathrm{~mm}$ y $0.075 \mathrm{~mm}$ se obtienen valores muy similares. Considerando el costo computacional, se optó por utilizar malla con tamaño de elemento $0.1 \mathrm{~mm}$ en todos los análisis posteriores.

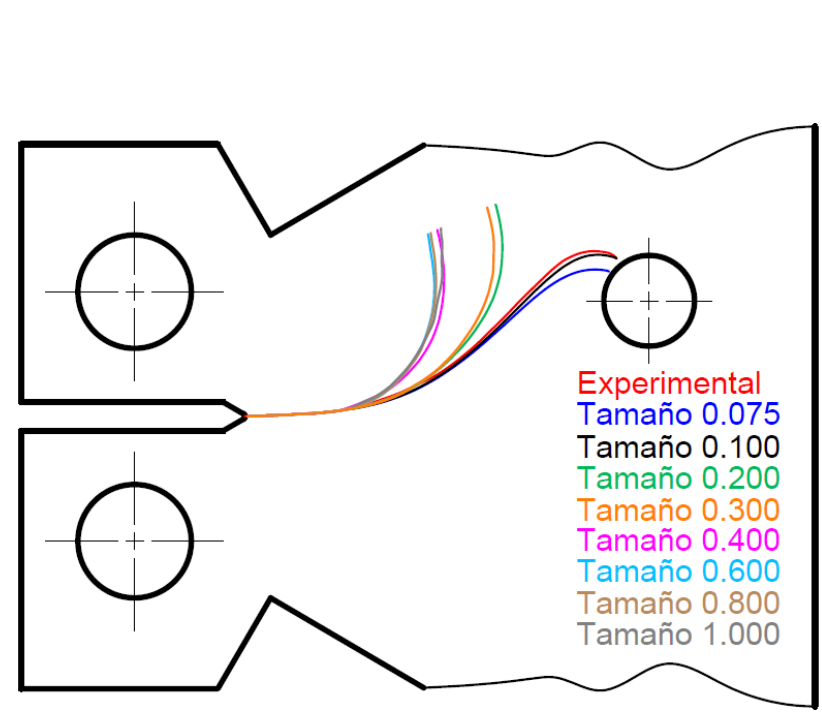

a) Resultados de la trayectoria

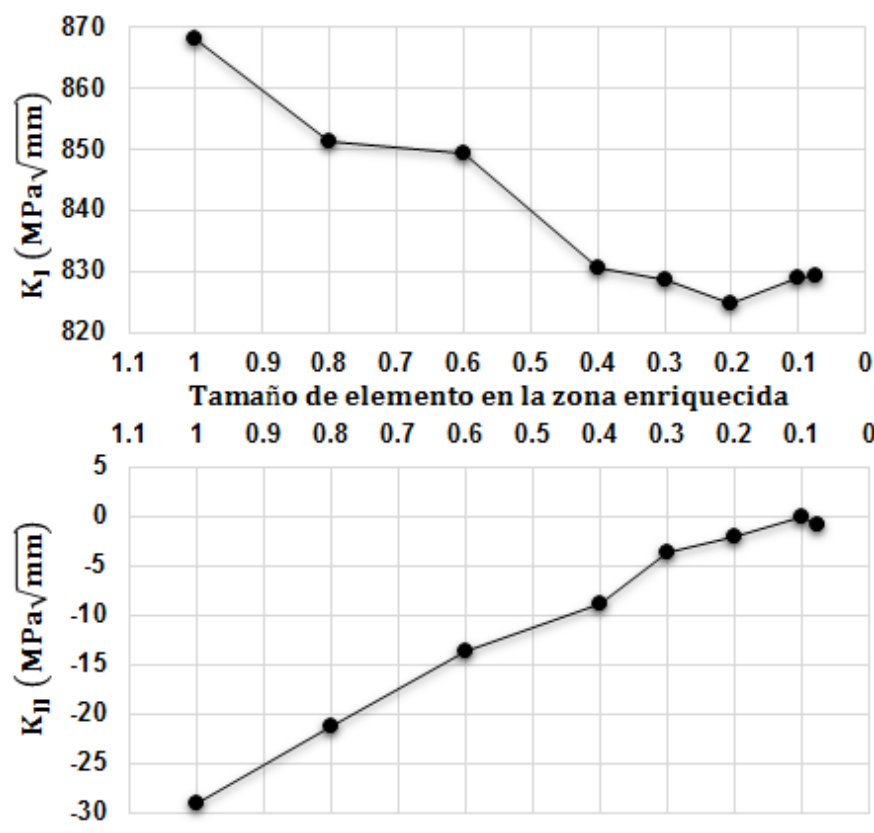

b) Valores de $K_{I}$ y $K_{I I}$

Fig. 5: Análisis de independencia de malla

Los tres criterios implementados para determinar la dirección de propagación fueron comparados igualmente en la probeta 3. En la Fig. 6 se muestra la trayectoria del resultado experimental (color rojo), y los resultados numéricos obtenidos por los autores usando los criterios MTS (color azul), MERR (color negro) y SED (color verde). Los resultados muestran que los tres criterios implementados ofrecen soluciones muy similares, presentándose prácticamente una superposición entre las trayectorias, como se observa en el detalle A de la Fig. 6. Estos resultados son corroborados cuantitativamente en la Tabla 4, en la que se muestra la diferencia máxima de la coordenada $Y_{T}$ de la trayectoria, entre los tres criterios y los resultados experimentales, indicándose la coordenada $X_{T}$ para la cual ocurre dicha diferencia.

En los puntos analizados las curvas son efectivamente coincidentes, obteniéndose un coeficiente de determinación $R^{2}$ de 0.997 , igual para los tres criterios, referido al resultado experimental.
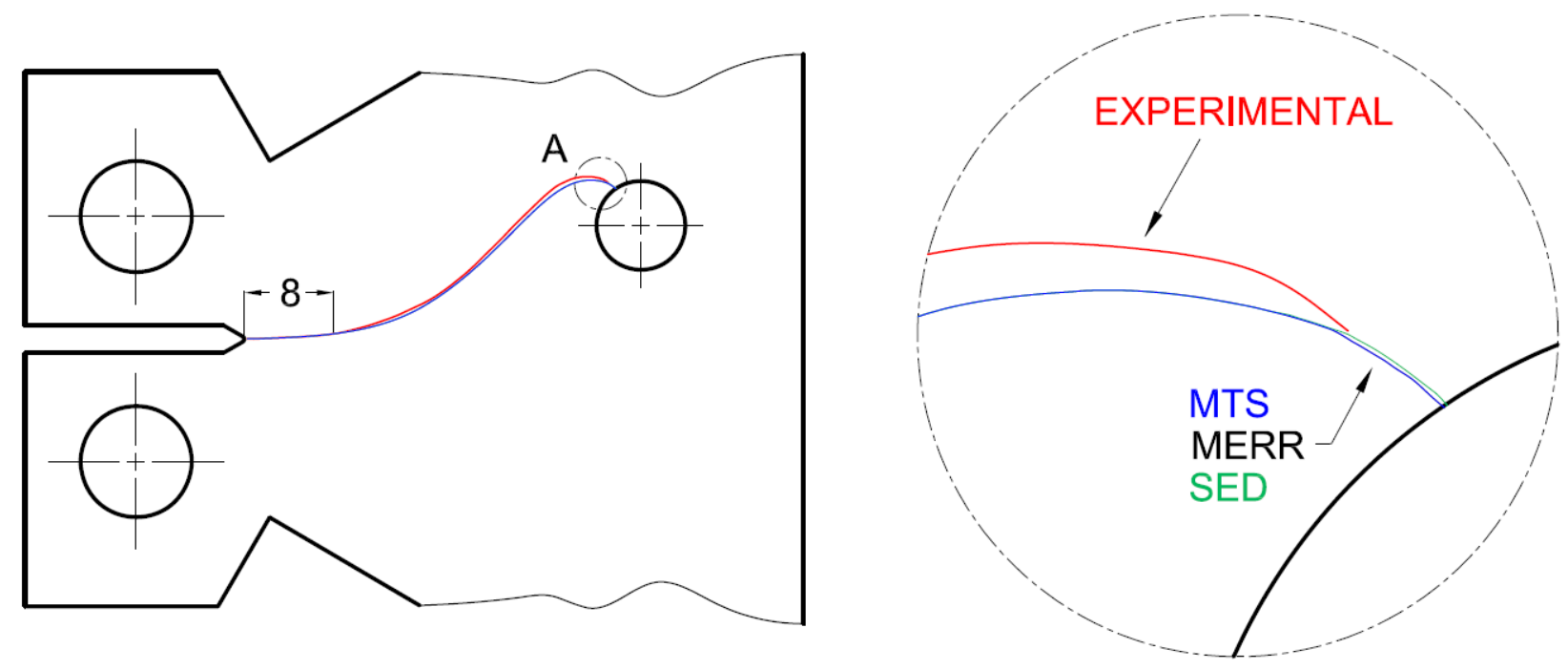

Detalle A

Fig. 6: Resultados cualitativos en la probeta 3 utilizando los criterios MTS, MERR y SED 
Tabla 4: Resultados cuantitativos en la probeta 3 utilizando los criterios MTS, MERR y SED

\begin{tabular}{|c|c|c|c|c|c|}
\hline & $X_{T}(\mathrm{~mm})$ & Experimental $Y_{T}(\mathrm{~mm})$ & Numérico $Y_{T}(\mathrm{~mm})$ & Diferencia máxima $\Delta Y_{T}(\mathrm{~mm})$ & $R^{2}$ \\
\hline MTS & 37.20 & 13.27 & 12.74 & 0.53 & 0.997 \\
\hline MERR & 37.20 & 13.27 & 12.74 & 0.53 & 0.997 \\
\hline SED & 37.20 & 13.27 & 12.74 & 0.53 & 0.997 \\
\hline
\end{tabular}

\section{Análisis de los resultados en las cinco probetas}

Los resultados numéricos se obtuvieron para los cinco casos de estudio utilizando los tres criterios para determinar la dirección de propagación de la fisura. Sin embargo, con el objetivo de comparar los resultados numéricos con los experimentales, se seleccionó el criterio MERR, pero pudo ser cualquiera de los tres. En la Tabla 5 se muestran los resultados cualitativos experimentales y numéricos de la trayectoria de la fisura.

Tabla 5: Resultados cualitativos en las cinco probetas utilizando el criterio MERR

\section{Foto de la probeta ensayada}
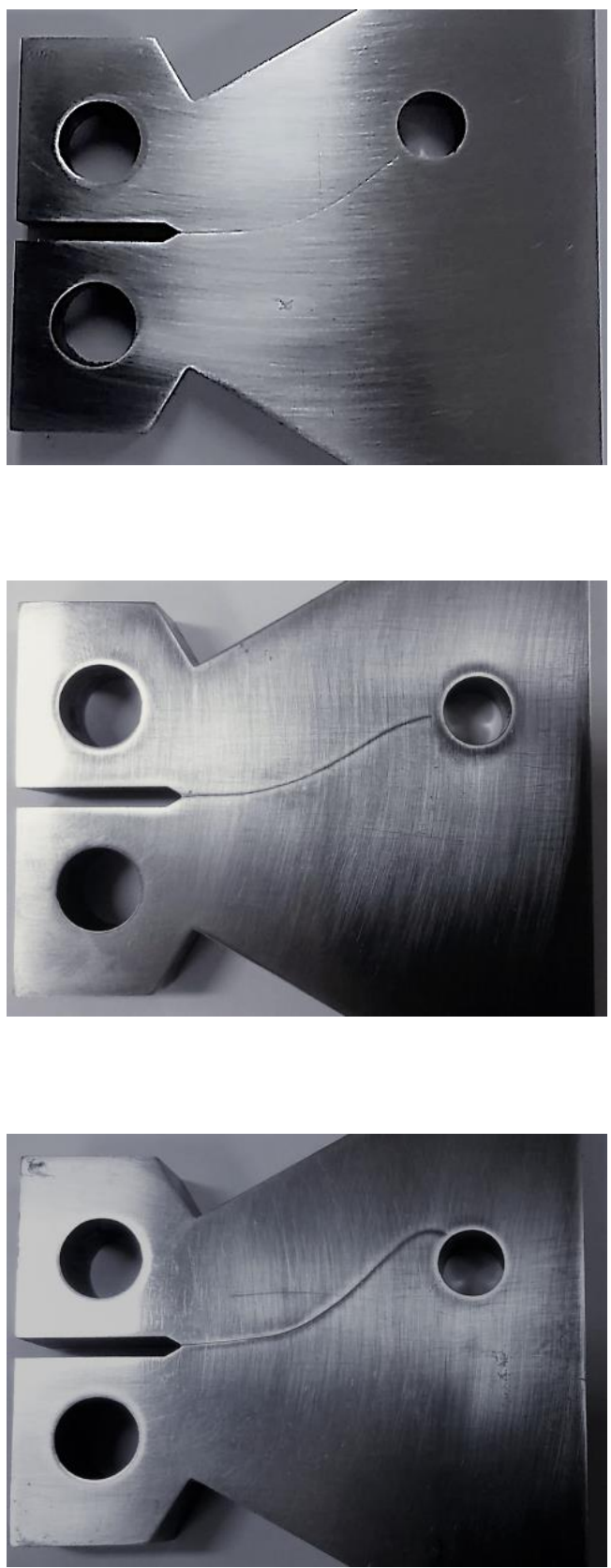

\section{Resultado experimental (rojo) y numérico (negro)}

Probeta 1

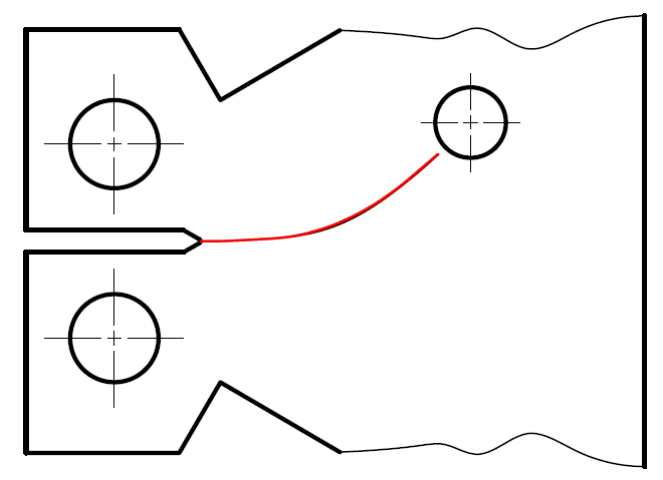

Probeta 2

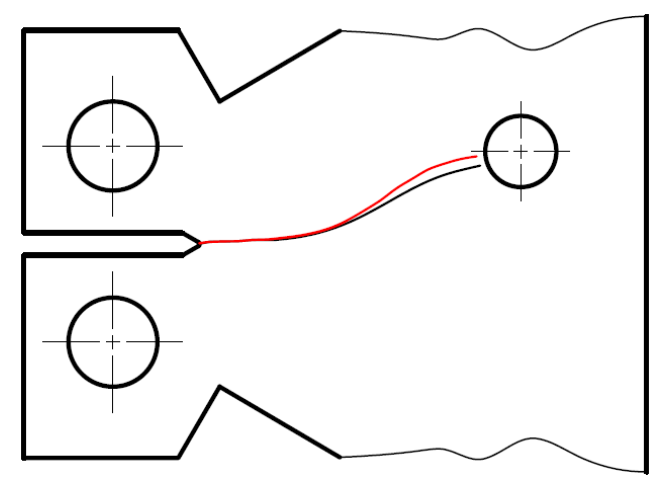

Probeta 3

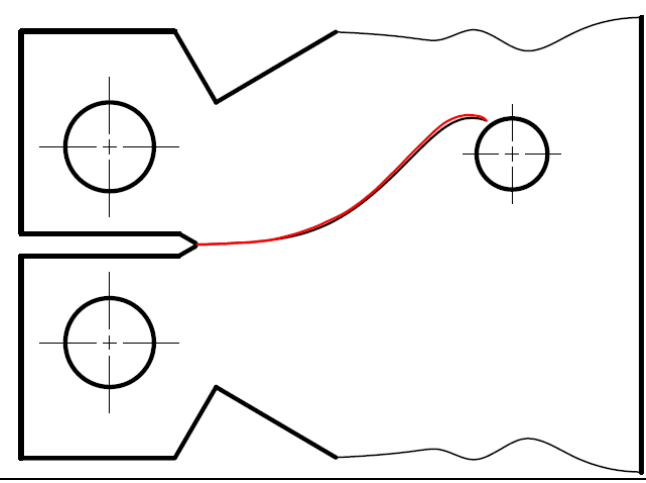


Tabla 5 (continuación)

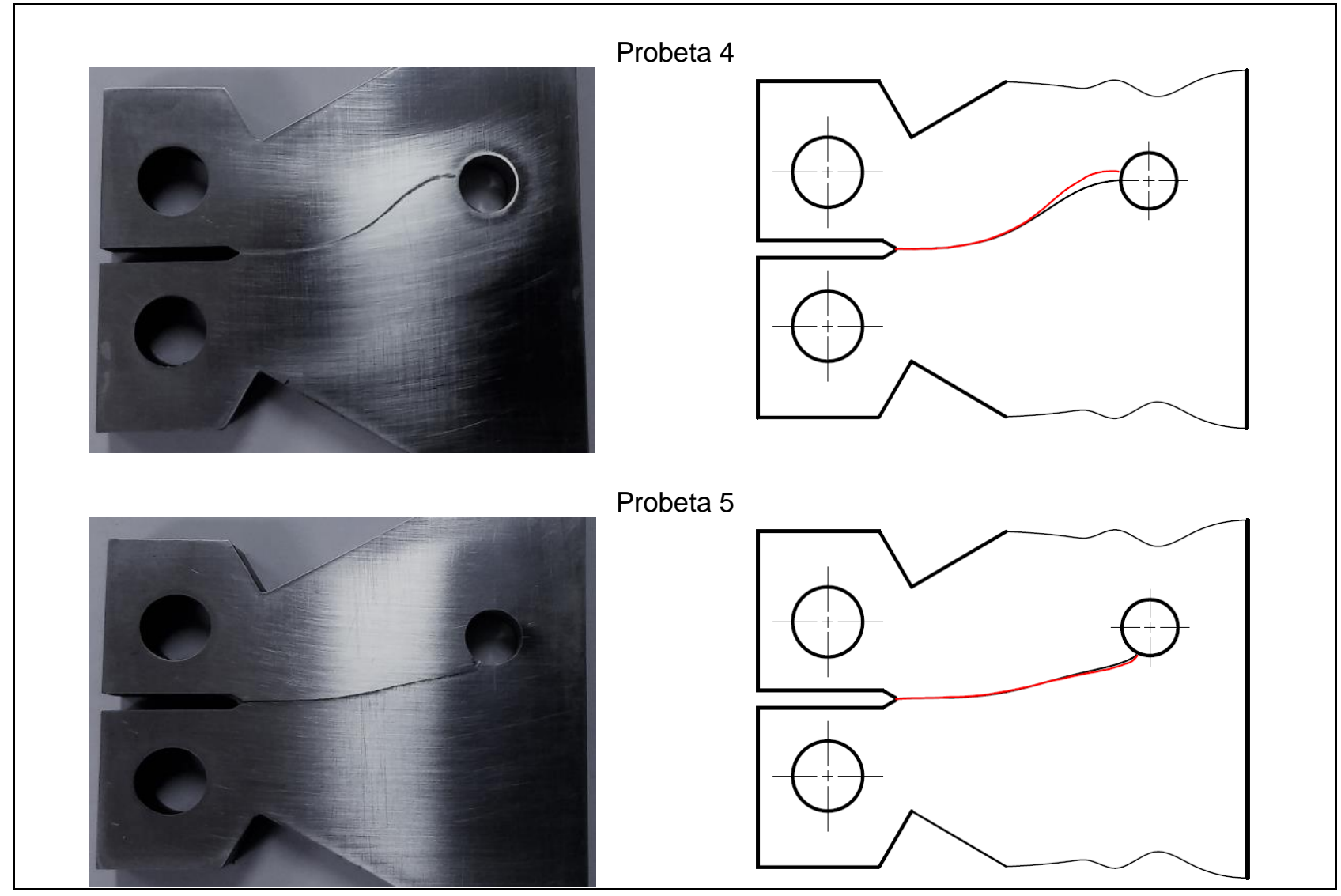

En la columna izquierda de la Tabla 5 aparece la foto de la probeta después del ensayo de propagación correspondiente, en la que se puede observar la trayectoria de la fisura propagada, mientras que en la columna derecha se ha dibujado la probeta y trazado las trayectorias obtenidas experimentalmente (color rojo) y numéricamente, según criterio MERR (color negro).

La Tabla 6 recoge los resultados cuantitativos para las cinco probetas analizadas. En ella se muestra la diferencia máxima de la coordenada $Y_{T}$ de la trayectoria, utilizando el criterio MERR y los resultados experimentales, indicándose la coordenada $X_{T}$ para la cual ocurre dicha diferencia. Se muestran también los coeficientes de determinación $R^{2}$, del resultado numérico respecto al experimental.

Tabla 6: Resultados cuantitativos en las cinco probetas utilizando el criterio MERR

\begin{tabular}{|c|c|c|c|c|c|}
\hline & $X_{T}(\mathrm{~mm})$ & Experimental $Y_{T}(\mathrm{~mm})$ & Numérico $Y_{T}(\mathrm{~mm})$ & Diferencia máxima $\Delta Y_{T}(\mathrm{~mm})$ & $R^{2}$ \\
\hline Probeta 1 & 26.8 & 2.76 & 2.56 & 0.20 & 0.9928 \\
\hline Probeta 2 & 35.6 & 8.44 & 7.13 & 1.31 & 0.9428 \\
\hline Probeta 3 & 37.2 & 13.27 & 12.74 & 0.53 & 0.9970 \\
\hline Probeta 4 & 38.0 & 10.75 & 9.17 & 1.58 & 0.9552 \\
\hline Probeta 5 & 43.6 & 5.50 & 6.64 & 1.14 & 0.9703 \\
\hline
\end{tabular}

Los resultados numéricos en los cinco casos de estudio muestran una fuerte concordancia con los resultados experimentales. La trayectoria de propagación de la fisura en las probetas 2 y 4, obtenida por simulación numérica, muestra gran coincidencia con la obtenida experimentalmente en la primera mitad y luego se va separando ligeramente, aunque siempre manteniendo la misma tendencia. Las diferencias entre la trayectoria obtenida numéricamente y experimentalmente pueden atribuirse a varios factores. Por ejemplo, las irregularidades micro estructurales del material, como son las inclusiones, los límites de granos, y los planos de deslizamiento, podrían desviar la trayectoria. Se debe tener en cuenta que en la simulación se considera un material ideal, que no contempla estos aspectos micro estructurales. Por otro lado, se comprobó que la dirección de propagación $\theta$ es muy sensible ante los valores calculados de $K_{I}$ y $K_{I I}$, que arrastran implícitamente errores de precisión y aproximación, ocasionando igualmente desviaciones en la trayectoria. 
A diferencia de lo anterior, en las probetas 1, 3 y 5 la correspondencia entre los resultados numéricos y experimentales se da a lo largo de toda la trayectoria. Estos resultados cualitativos están en correspondencia con los resultados cuantitativos del coeficiente de determinación $R^{2}$, que muestra valores más altos para las probetas 1,3 y 5 y más bajos para las probetas 2 y 4 .

La influencia del taladro desviador en la trayectoria de la fisura es claramente captada en las cinco probetas analizadas. Se encontró que la pre-fisura inicial también influye en la trayectoria de propagación. En la probeta 5 la pre-fisura no tuvo desviación inicial considerable, por lo que la propagación mantuvo una tendencia horizontal en la mayor parte de la trayectoria, captando la influencia ejercida por el taladro en el tramo final.

\section{CONCLUSIONES}

Es de gran importancia establecer procedimientos para realizar los ensayos experimentales de propagación de fisuras por fatiga, ya que no existen normas técnicas para este fin. Los resultados de las trayectorias, obtenidos experimentalmente, demuestran que el diseño propuesto y el proceso de fabricación de las probetas son adecuados. En tal sentido, los autores consideran que el presente estudio constituye un aporte para la investigación en el campo de la mecánica de la fractura.

El procedimiento numérico desarrollado e implementado para simular la trayectoria de propagación de la fisura, usando tecnología XFEM y el método LSM, permitió predecir con alta precisión la trayectoria de propagación de fisuras por fatiga. La experiencia en el desarrollo de las rutinas, para aplicar los criterios que determinan la dirección de propagación de la trayectoria de la fisura, no sólo permite usar los criterios MTS, MERR y SED, sino que permitiría evaluar cualquier otro criterio. Esto es útil para investigaciones futuras, en las que se desee profundizar en los criterios de propagación. Los tres criterios implementados arrojan resultados prácticamente idénticos, alcanzando un coeficiente de determinación $R^{2}$ igual a 0.997 , respecto a los resultados experimentales en la probeta 3. En este procedimiento los autores detectaron un posible error en la simulación, cuando la fisura tiende a cortar dos veces a un mismo elemento, debido a que el método LSM está limitado a cortar una sola vez cada elemento. Se propuso una solución que permite corregir el valor del ángulo y continuar la simulación, obteniéndose buenos resultados.

Se encontró una fuerte correspondencia entre los resultados numéricos y experimentales en todos los casos de estudio, obteniéndose coeficientes de determinación $R^{2}$ que oscilan entre 0.9428 y 0.9970 . Esta correspondencia permite concluir que el taladro desviador tiene gran influencia en la trayectoria de propagación de la fisura. Desde el punto de vista de la simulación numérica, los parámetros más influyentes en la trayectoria de propagación de la fisura, en el campo de la LEFM, son los factores de intensidad de tensiones $K_{I}$ y $K_{I I}$, por lo que resulta de interés continuar investigando en la determinación precisa de sus valores.

Los resultados demuestran que las probetas tipo doble voladizo TDCB, propuestas para los ensayos de propagación de fisuras, incluyendo un taladro desviador, permiten obtener mayor cantidad de datos experimentales, así como trayectorias más largas y complejas, donde se producen modos de carga combinada I+II. Además, el uso de la técnica XFEM evita la necesidad de remallado cuando la fisura propaga por el elemento y facilita la obtención de $K_{I}$ y $K_{I I}$. Estas dificultades han sido encontradas en investigaciones como las desarrolladas por Silva et al. (2017), donde utilizan probetas CT para los ensayos experimentales y el FEM en la simulación de propagación de fisuras.

Los procedimientos desarrollados en el presente estudio están enmarcados en el campo de la LEFM y son adecuados para obtener la trayectoria de propagación de fisuras sometidas a cargas cíclicas y de amplitud constante. La validez de los resultados está limitada a materiales con comportamiento frágil, tales como cerámicos y aceros fragilizados por tratamientos térmicos o que trabajan a temperaturas inferiores a la temperatura de transición. Se pueden analizar componentes fisurados en 2D, con la posibilidad de considerar estados de tensión plana o de deformación plana.

\section{AGRADECIMIENTOS}

A la Dirección de Gestión de la Investigación de la PUCP, al Grupo de Investigación Asistida por Computadora (INACOM/Aula PUCP-CIMNE) de la Sección Ingeniería Mecánica del Departamento de Ingeniería de la PUCP, al Programa Ingeniería de la Soldadura (INGESOLD) de la PUCP y al Laboratorio de Materiales de la Sección Ingeniería Mecánica del Departamento de Ingeniería de la PUCP, por brindar todo el apoyo para desarrollar la presente investigación. 


\section{REFERENCIAS}

Andrade, A.A., W.A. Mosquera y L.V. Vanegas, Modelos de Crecimiento de Grietas por Fatiga, ISSN: 1909-8367, Entre Ciencia e Ingeniería, 18, 39-48 (2015)

Ayatollahi, M.R., S.M.J. Razavi e Y. Yahya, Mixed Mode Fatigue Crack Initiation and Growth in a CT Specimen Repaired by Stop Hole Technique, doi: 10.1016/j.engfracmech.2015.03.027, Eng. Fract. Mech., 145, 115-127 (2015)

ASTM A370-17, Standard Test Methods and Definitions for Mechanical Testing of Steel Products1, doi: 10.1520/A037017 (2017)

ASTM E18-16, Standard Test Methods for Rockwell Hardness of Metallic Materials1, 2, doi: 10.1520/E0018-16 (2016)

ASTM E399-12, Standard Test Method for Linear-Elastic Plane-Strain Fracture Toughness KIC of Metallic Materials1, doi: 10.1520/E0399 (2013)

Bahloul, A., CH. Bouraoui y T. Boukharouba, Prediction of fatigue life by crack growth analysis, doi: 10.1007/s00170-0170069-8, Int. J. Adv. Manuf. Technol., 91(9-12), 4009-4017 (2017)

Bergara, A., J. I. Dorado, A. Martín-Meizoso y J. M. Martínez-Esnaola, Fatigue crack propagation in complex stress fields: experiments and numerical simulations using the Extended Finite Element Method (XFEM), doi: 10.1016/j.ijfatigue.2017.05.026, Int. J. Fatigue, 103, 112-121 (2017)

Bouchard, P.O., F. Bay e Y. Chastel, Numerical Modelling of Crack Propagation: Automatic Remeshing and Comparison of Different Criteria, doi: 10.1016/S0045-7825(03)00391-8, Comp. Methods Appl. Mech. Eng., 192, 3887-3908 (2003)

Branco, R., F.V. Antunes y J.D. Costa, A review on 3D-FE Adaptive remeshing techniques for crack growth modelling, doi: 10.1016/j.engfracmech.2015.05.023, Eng. Fract. Mech., 141, 170-195 (2015)

Cotterell, B. y J.R. Rice, Slightly Curved or Kinked Cracks, doi: 10.1007/BF00012619, Int. J. Fract., 16(2), 155-169 (1980)

Erdogan, F. y G.C. Sih, On the Crack Extension in Plates under Plane Loading and Transverse Shear, doi: 10.1115/1.3656897, J. Basic Eng., 85(4), 519-525 (1963)

Fernández, M. y N. Domínguez, Nuevos Horizontes en el Estudio de Propagación de Grietas en Estructuras por medio del Método XFEM, Memorias de XVII Congreso Nacional de Ingeniería Estructural, 1-22, León, México, 3 al 6 de Noviembre (2010)

Ferreño, D., J.M. Alegre y J.M. Revilla, Simulación del Efecto de la Plastificación en la Propagación de Fisuras por Fatiga en Modo Mixto, Anales de Mecánica de la Fractura, XXIII Encuentro del Grupo Español de Fractura, 1, 275-280, Albarracín, España del 29 a 31 Marzo (2006)

Gonzáles, O.A., Estimación y Acotación del Error de Discretización en el Modelado de Grietas mediante el Método Extendido de los Elementos Finitos, Tesis de Doctorado, Dpto. de Ingeniería Mecánica y de Materiales, Universidad Politécnica de Valencia, Valencia, España (2010)

Griffith, A.A., The Phenomena of Rupture and Flow in Solids, doi: 10.1098/rsta.1921.0006, Phil. Trans. R. Soc. Lond. A, 221, 163-198 (1921)

Hussain, M.A., S.L. Pu y J. Underwood, Strain Energy Release Rate for a Crack under Combined Mode I and Mode II, doi: 10.1520/STP33130S, ASTM STP 560, 2-28 (1974)

Irwin, G.R., Analysis of Stresses and Strains Near the end of Crack Traversing a Plate, J. Appl. Mech., 24, 351-369 (1957)

Islam, M.A., Critical Assessment of the Degree of Temper Embrittlement in 2.25Cr-1Mo Steel, ISSN: 1819-6608, ARPN J. Eng. Appl. Sci., 1(1), 39-45 (2006)

Martins, R.F., L. Ferreira, L. Reis y P. Chambel, Fatigue Crack Growth under Cyclic Torsional Loading, doi: 10.1016/j.tafmec.2016.08.016, Theo. App. Fract. Mech., 85, 56-66 (2016)

Moës, N., J. Dolbow y T. Belytschko, A Finite Element Method for Crack Growth without Remeshing, doi: 10.1002/(SICI)10970207(19990910)46:1<131::AID-NME726>3.0.CO;2-J, Int. J. Numer. Methods Eng., 46(1), 131-150 (1999)

Mróz, K.P. y Z. Mróz, On Crack Path Evolution Rules, doi: 10.1016/j.engfracmech.2010.03.038, Eng. Fract. Mech., 77, 1781-1807 (2010)

Palaniswamy, K. y W.G. Knauss, On the Problem of Crack Extension in Brittle Solids under General Loading, doi: 10.1016/B978-0-08-021792-5.50010-0, Mechanics Today, 4, 87-148 (1978)

Palma, J.A., J.A. Barbosa y A.A. Silva, Simulación Numérica de la Propagación de una Fisura en un Material Degradado por efecto de la Fragilización por Hidrogeno, doi: 10.1612/inf.tecnol.4161it.09, Inf. Tecnol., 21(2), 65-76 (2010)

Pathak, H., A. Singh y I.V. Singh, Fatigue Crack Crowth of 3-D Problems using XFEM, doi: 10.1016/j.jjmecsci.2013.09.001, Int. J. Mechanical Sciences, 76, 112-131 (2013)

Richard, H.A., B. Schramn y N.H. Schirmeisen, Crack on Mixed Mode Loading-Theories, Experiments, Simulations, doi: 10.1016/j.ijfatigue.2013.06.019, Int. J. Fatigue, 62, 93-103 (2013)

Shariati, M., E. Mohammadi y R.M. Nejad, Effect of a New Specimen Size on Fatigue Crack Growth Behavior in ThickWalled Pressure Vessels, doi: 10.1016/j.ijpvp.2016.12.009, Int. J. Press. Vessels Pip., 150, 1-10 (2017) 
Sharma, K., S. Bhattacharya y V. Sonkar, XFEM Simulation on Mixed-Mode Fatigue Crack Growth of Functionally Graded Material, J. Mech. Eng. and Biomechanics, 1(1), 46-55 (2016)

Silva, A.L.L., A.M.P. de Jesus y otros tres autores, Combined Analytical-Numerical Methodologies for the Evaluation of Mixed-Mode (I + II) Fatigue Crack Growth Rates in Structural Steels, doi: 10.1016/j.engfracmech.2017.04.016, Eng. Fract. Mech., 185, 124-138 (2017)

Stolarska, M, D.L. Chopp, N. Moës y T. Belytschko, Modelling Crack Growth by Level Sets in the Extended Finite Element Method, doi: 10.1002/nme.201, Int. J. Numer. Methods Eng., 51, 943-960 (2001)

Sih, G.C., Strain-Energy-Density Factor Applied to Mixed Mode Crack Problems, doi: 10.1007/BF00035493, Int. J. Fract., 10(3), 305-321 (1974)

Sih, G.C. y B. Macdonald, Fracture Mechanics Applied to Engineering Problems-Strain Energy Density Fracture Criterion, doi: 10.1016/0013-7944(74)90033-2, Eng. Fract. Mech., 6(2), 361-386 (1974)

Wu, C.H., Fracture under Combined Loads by Maximum-Energy-Release-Rate Criterion, doi: 10.1115/1.3424360, J. Appl. Mech., 45(3), 553-558 (1978 a)

Wu, C.H., Maximum-Energy-Release-Rate Criterion Applied to a Tensión-Compressión Specimen with Crack, doi: 10.1007/BF00130464, J. Elast., 8(3), 235-257 (1978 b)

Wang, Y., G. Xie, Z. Zhang, X. Qian, Y. Zhou y J. Li, Effect of Carbide Content on Temper Embrittlement of 2.25Cr1Mo Steel, doi:10.1115/PVP2016-63289, ASME Digital Collection, ASME 2016 PVP Conference, 1, 1-8, (2016) 